\title{
Aqueous Stable Gold Nanostar/ZIF-8 Nanocomposites for Light-Triggered Release of Active Cargo Inside Living Cells
}

Carolina Carrillo-Carrión, Raquel Martínez, María F. Navarro Poupard, Beatriz Pelaz, Ester Polo, Ana Arenas-Vivo, Alessandro Olgiati, Pablo Taboada, Mahmoud G. Soliman, Úrsula Catalán, Sara Fernández-Castillejo, Rosa Solà, Wolfgang J. Parak, Patricia Horcajada, Ramon A. Alvarez-Puebla and Pablo del Pino

\section{Peer reviewed version}

This is the peer reviewed version of the following article: C. Carrillo-Carrión, R. Martínez, M. F. Navarro Poupard, B. Pelaz, E. Polo, A. Arenas-Vivo, A. Olgiati, P. Taboada, M. G. Soliman, Ú. Catalán, S. Fernández-Castillejo, R. Solà, W. J. Parak, P. Horcajada, R. A. Alvarez-Puebla, P. del Pino, Angew. Chem. Int. Ed. 2019, 58, 7078, which has been published in final form at https:// doi.org/10.1002/anie.201902817. This article may be used for non-commercial purposes in accordance with Wiley Terms and Conditions for Use of Self-Archived Versions

\section{How to cite:}

C. Carrillo-Carrión, R. Martínez, M. F. Navarro Poupard, B. Pelaz, E. Polo, A. Arenas-Vivo, A. Olgiati, P. Taboada, M. G. Soliman, Ú. Catalán, S. Fernández-Castillejo, R. Solà, W. J. Parak, P. Horcajada, R. A. Alvarez-Puebla, P. del Pino, Angew. Chem. Int. Ed. 2019, 58, 7078

\section{Copyright information:}

(C) 2019 WILEY-VCH Verlag GmbH \& Co. KGaA, Weinheim. This article may be used for non-commercial purposes in accordance with Wiley Terms and Conditions for Use of SelfArchived Versions 


\title{
Aqueous Stable Gold Nanostar/ZIF-8 Nanocomposites for Light Triggered Release of Active Cargo Inside Living Cells
}

\author{
Carolina Carrillo-Carrión ${ }^{\dagger}$, Raquel Martínez ${ }^{\dagger}$, María F. Navarro Poupard, Beatriz Pelaz, Ester Polo, Ana \\ Arenas-Vivo, Alessandro Olgiati, Pablo Taboada, Mahmoud G. Soliman, Úrsula Catalán, Sara \\ Fernández-Castillejo, Rosa Solà, Wolfgang J. Parak, Patricia Horcajada, ${ }^{*}$ Ramon A. Alvarez-Puebla, ${ }^{*}$ \\ and Pablo del Pino*
}

\begin{abstract}
A plasmonic core-shell gold nanostars/zeolitic imidazolate framework-8 (ZIF-8) nanocomposite was developed for thermoplasmonic-driven release of encapsulated active molecules inside living cells. The nanocomposites were loaded, as a proof of concept, with bisbenzimide molecules as functional cargo, and wrapped with an amphiphilic polymer that prevents ZIF-8 degradation and bisbenzimide leaking in aqueous media or inside living cells. The demonstrated molecule-release mechanism relies on the use of nearIR light coupled to the plasmonic absorption of the core gold nanostars, which creates local temperature gradients and thus, bisbenzimide thermodiffusion. Confocal microscopy and surfaceenhanced Raman spectroscopy (SERS) were used to demonstrate bisbenzimide loading/leaking and near-IR-triggered cargo release inside cells, thereby leading to DNA staining.
\end{abstract}

Metal-organic-frameworks (MOFs), consisting in cations coordinated with multidentate organic ligands, ${ }^{[1]}$ have recently emerged as the latest class of promising drug nanocarriers. ${ }^{[2]}$ The MOF most basic physicochemical properties (that is, porosity, hydrophilicity/hydrophobicity, biostability) are key parameters to

$\left.{ }^{*}\right] \quad$ Dr. C. Carrillo-Carrión ${ }^{[+]}$, R. Martínez ${ }^{[+]}$, M. F. Navarro Poupard, Dr. B. Pelaz, Dr. E. Polo, A. Olgiati, Dr. P. del Pino

Centro Singular de Investigación en Química Biolóxica e Materiais Moleculares (CiQUS), Departamento de Física de Partículas, Universidade de Santiago de Compostela, Santiago de Compostela (Spain)

E-mail: pablo.delpino@usc.es

A. Arenas-Vivo, Dr. P. Horcajada

Advanced Porous Materials Unit, IMDEA Energy, Madrid (Spain)

E-mail: patricia.horcajada@imdea.org

Dr. P. Taboada

Departamento de Física de Partículas, Universidade de Santiago de

Compostela, Santiago de Compostela (Spain)

Dr. M. G. Soliman, Prof. W. J. Parak

Fachbereich Physik and CHyN, Universität Hamburg, Hamburg, (Germany)

Dr. M. G. Soliman

Physics Department, Faculty of Science, Al-Azhar University, Cairo (Egypt)

Dr. Ú. Catalán, S. Fernández-Castillejo, Prof. R. Solà

Facultat de Medicina i Ciències de la Salut, Universitat Rovira i Virgili,

Reus (Spain)

Dr. Ú. Catalán

Institut d'Investigació Sanitaria Pere Virgili (IISPV), Reus (Spain)

Prof. R. Solà

Hospital Universitari Sant Joan de Reus, Reus (Spain)

Prof. R. A. Alvarez-Puebla

Departamento de Química Física e Inorgánica and EMaS, Universitat

Rovira i Virgili, Tarragona (Spain)

ICREA, Barcelona (Spain)

E-mail: ramon.alvarez@urv.cat

['] These authors contributed equally to this work.

Supporting information for this article and the ORCID identification number(s) for the author(s) of this article can be found under $\mathrm{xxxx}$ fabricate efficient drug-delivery systems, combining high drug loadings with a controlled release. Despite their associated exceptional cargoes, the often limited aqueous (bio)stability of MOFs limits their preclinical application. As a representative example, the biocompatible microporous zeolitic imidazolate framework 8 (ZIF-8) $)^{[3]}$ have been widely proposed for sustained delivery of wide variety of challenging drugs. ${ }^{[4-7]}$ Although with progressive release, most previous reports refer to that $80-90 \%$ of the encapsulated cargo is released within the first $\sim 10 \mathrm{~h}$ after exposure to physiological environments. These properties are derived from the chemical instability of ZIF-8 in water, ${ }^{\left[{ }^{[8]}\right.}$ which degrades depending on the ZIF-8 mass to water ratio and medium $\mathrm{pH},{ }^{[5,9]}$ thus releasing its cargo.

In this context, nanocomposites based on MOF and inorganic nanoparticles (NPs) seem a good strategy to promote drug delivery under a given stimulus (e.g., light, magnetic fields, ultrasounds), as already demonstrated for other materials. ${ }^{[10]}$ These type of nanocomposites have been recently explored for optical sensing, ${ }^{[11]}$ therapeutics, ${ }^{[12]}$ theragnostic, ${ }^{[13]}$ and heterogeneous catalysis with encouraging results. ${ }^{[14]}$ Nevertheless, the precise (temporal and spatial) controlled delivery of the cargo inside cells upon stimuli has not been achieved, since the challenging inherent aqueous instability of MOFs has not yet been addressed.

In this communication, we designed a thermoresponsive carrier comprising gold nanostars (NSs) coated with ZIF-8 stabilized with the amphiphilic polymer poly[isobutylene-altmaleic anhydride]-graft-dodecyl (PMA). The newly developed nanocomposite (NC) has high drug loading (similar to the conventional ZIF-8), is stable in aqueous solution and can be activated to release the cargo upon illumination with near-IR (NIR) light. This concept is demonstrated both in aqueous solution and inside living cells.

NSs were selected as core due to their characteristic plasmonic band centred $\sim 770 \mathrm{~nm}$ (Figure S1), ${ }^{[15]}$ which is suitable for excitation within the first biological window. ${ }^{[16]}$ These NSs were used as seeds to grow the ZIF-8 shell. ${ }^{[11,17]}$ The number of ZIF8's seeds was optimized to minimize byproducts such as noncoated NSs and/or pristine ZIF-8 NPs. The resulting NCs showed polyhedral morphology with average size of $218 \pm 24 \mathrm{~nm}$ (Figure $1 \mathrm{~A}-\mathrm{C}$ and Figure S2). As bioactive cargo/reporter we choose the bisbenzimide compound Hoechst H 33258 (HOE), a blue fluorescent dye typically used for DNA staining in molecular biology. ${ }^{[18]}$ The properties of HOE are particularly interesting for our purposes because: DNA-unbound HOE is less fluorescent (ca. $1: 30)$ and emits at a longer wavelength ( $520 \mathrm{vs} .461 \mathrm{~nm})$ than the DNA-bound one; and free or released from the carrier HOE will stain cell nuclei. ${ }^{[18]} \mathrm{NCs}$ were loaded with $\mathrm{HOE}$ (NC-HOE) by immersion of the NCs in a HOE solution (methanol). After 
purification, an average of $\sim 2.9 \times 10^{5} \mathrm{HOE} / \mathrm{NC}$ was estimated by fluorescence measurements (Figure S3).

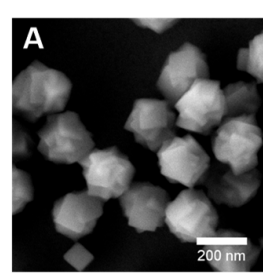

D

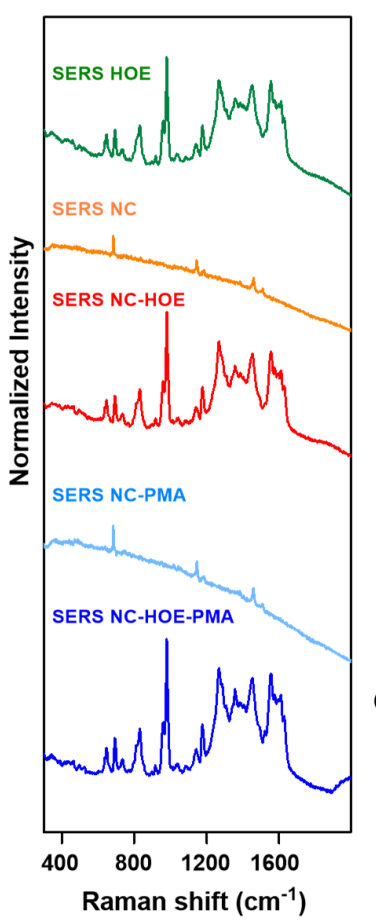

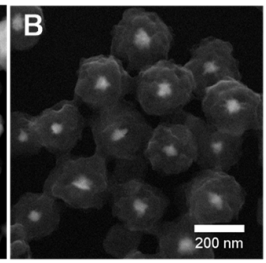
E 西
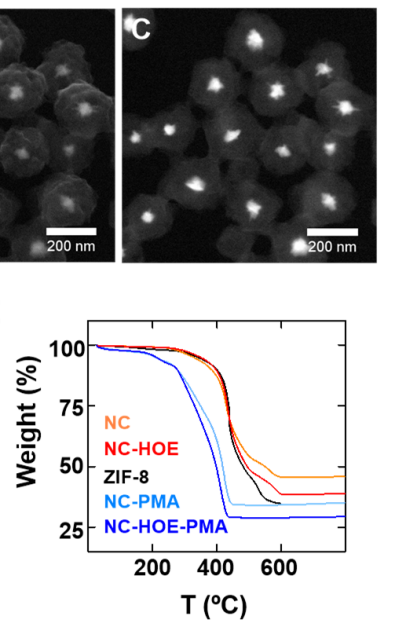

F

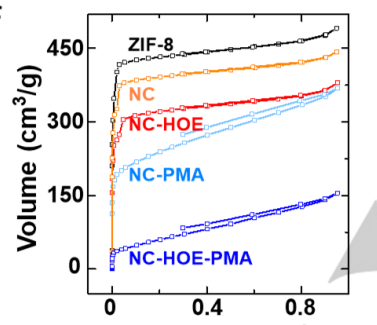

Relative pressure $\left(\mathrm{P} / \mathrm{P}_{0}\right)$

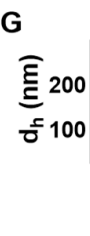

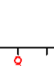

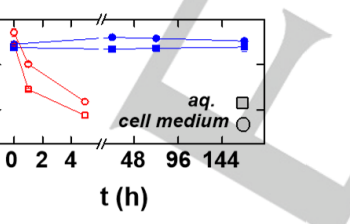

Figure 1. A-C) SEM micrographs of NCs; images acquired at different voltages and with different detectors: (A) Everhart-Thornley detector (SE2, secondary electrons) at $3 \mathrm{kV},(\mathrm{B}) \mathrm{InLens}$ detector (SE1, secondary electrons) at $20 \mathrm{kV}$, and (C) AsB detector (backscattered electrons) at $20 \mathrm{kV}$. D) SERS spectra of free HOE and the NCs with or without HOE trapped: HOE, NC and NC-HOE in methanol, and NC-PMA and NC-HOE-PMA in water. E) TGA of the different NCs. F) $\mathrm{N}_{2}$ sorption isotherms at $77 \mathrm{~K}$ of the different NCs. G) Mean dh during one week for the NC (red) and NC-PMA (blue) systems in water (squares) and cell medium (circles).

Next, a polymer grafting strategy was developed to "trap" the HOE inside the ZIF-8 voids. To that aim, we employed a postfunctionalization strategy based on PMA (Figure S4), which has been extensively used for polymer coating of inorganic NPs. ${ }^{[15]}$ After PMA functionalization and redispersion in water, the purified nanocomposites (NC-HOE-PMA) retained the polyhedral morphology and size (Figure S5-S7), bluish aspect (color and turbidity, Figure $S 8)$ and the hydrodynamic diameter $\left(d_{h}=235 \pm\right.$ $85 \mathrm{~nm}$ by dynamic light scattering, DLS, Figure S9 and Table S1) of the original $\mathrm{NCs}$, with $\zeta$-potential $=-27.5 \mathrm{mV}$, consistent with that obtained from other PMA-coated NPs. ${ }^{[15]}$ Post PMA- functionalization, the HOE loading dropped to $\sim 40 \%$ of the original content $\left(\sim 1.2 \cdot 10^{5} \mathrm{HOE} / \mathrm{NC}\right)$, suggesting that some dyeleaking occurred during PMA-functionalization and/or PMA partially replaced the dye within the porosity. PMA and non-PMA samples exhibit the characteristic Bragg peaks of ZIF-8 and $\mathrm{Au}^{0}$, confirming that PMA-functionalization does not affect the ZIF-8 shell crystallinity (see powder X-ray diffraction (PXRD), Figure S10 and Table S2-S4).

To test the encapsulation of HOE into the MOF composites, we studied the SERS spectra of the different NCs (with and without PMA and/or HOE) and free HOE (Figure 1D). The plasmonic NCs without $\mathrm{HOE}$ were dominated by the characteristic vibrational modes of ZIF-8 (the imidazole ring puckering, $685 \mathrm{~cm}^{-}$ ${ }^{1}$; the $\mathrm{CN}$ stretching, $1146 \mathrm{~cm}^{-1}$ and the $\mathrm{CH}$ waging, $\left.1460 \mathrm{~cm}^{-1}\right) \cdot{ }^{[19]}$ Notably, when the NCs were loaded with HOE, none of these vibrational features can be observed as a consequence of the strong signal promoted by the dye in close contact with the plasmonic surface (ring breathing, $980 \mathrm{~cm}^{-1} ; \mathrm{CH}$ deformation 1270 and $1455 \mathrm{~cm}^{-1}$ and $\mathrm{CC}$ and $\mathrm{CN}$ stretchings, 1554 and 1575 $\mathrm{cm}^{-1}$ ).

To investigate the thermal behaviour of the prepared NCs and pristine ZIF-8, thermogravimetric analysis (TGA) was performed under air atmosphere (Figure 1E). The samples without PMA showed a sharp weight loss (50-60 wt\%) at around $400{ }^{\circ} \mathrm{C}$, associated with the total combustion of 2-methylimidazole and the formation of different inorganic residues: $\mathrm{ZnO}$ in case of ZIF-8, or $\mathrm{ZnO}$ and $\mathrm{Au}^{0}$ in case of NC and NC-HOE, as identified by XRPD of the residues (Table S5 and Figure S11). In contrast, the presence of PMA is evidenced by the more abrupt weight loss before $400{ }^{\circ} \mathrm{C}$ on PMA-modified samples. They showed an initial mass loss $(\sim 10 \mathrm{wt} \%)$ associated with the PMA glass transition $\left(150-250{ }^{\circ} \mathrm{C}\right)$ and final departure $(\sim 20 \mathrm{wt} \%)$ at around 250 $350^{\circ} \mathrm{C} \cdot{ }^{[15]}$ Finally, weight loss is followed by the combustion of the 2-methylimidazole as in non-PMA samples $\left(\sim 400{ }^{\circ} \mathrm{C}\right)$. The different inorganic residues served us to estimate the relative content of Au, PMA, ZIF-8 and HOE among samples (Table S5).

$\mathrm{N}_{2}$ sorption measurements at $77 \mathrm{~K}$ of ZIF-8, NC, NC-HOE, NCPMA and NC-HOE-PMA show type I isotherms (Figure 1F), characteristic of microporous materials, with a Brunauer, Emmett, and Teller specific surface areas $\left(\mathrm{S}_{\mathrm{BET}}\right)$ of 1800, 1570, 1260, 850 and $220 \mathrm{~m}^{2} \cdot \mathrm{g}^{-1}$, respectively. The normalization of the $\mathrm{S}_{\mathrm{BET}}$ by the porous ZIF-8 weight shows no significant difference between the ZIF-8 and NCs, indicating that the ZIF-8 shell shares the same textural properties as the pristine ZIF-8 (Table S6). Counter like, the important reduction of the microporosity (normalized $\mathrm{S}_{\mathrm{BET}}$ before and after HOE encapsulation $\sim 1800$ versus $1500 \mathrm{~m}^{2} \cdot \mathrm{g}^{-1}$ ) confirms that the HOE is allocated within the ZIF-8 shell. Importantly, corrected surfaces indicate that PMA, besides of providing an outer coating for the NPs, partially fills NC-PMA ( $\mathrm{S}_{\text {BET }}$ $\approx 1440 \mathrm{~m}^{2} \cdot \mathrm{g}^{-1}$ ), which is concordance with the results found by fluorescence $\left(\sim 60 \%\right.$ HOE loss after PMA, final corrected $\mathrm{S}_{\mathrm{BET}} \approx$ $470 \mathrm{~m}^{2} \cdot \mathrm{g}^{-1}$ ). Further, the additional and progressive $\mathrm{N}_{2}$ sorption at higher pressures $\left(P / P_{0}=0.3-0.9\right)$ in the PMA associated samples suggests the gas adsorption on the superficial polymer branches $\left(0.25 \mathrm{~cm}^{3} \cdot \mathrm{g}^{-1}\right.$ in NC-PMA versus $0.06 \mathrm{~cm}^{3} \cdot \mathrm{g}^{-1}$ in the pristine ZIF-8). Of note, the volume occupied by $\mathrm{HOE}$ in $\mathrm{NC}$ (estimated from the total $\mathrm{HOE}$ content and $\mathrm{N}_{2}$ sorption measurements: $V \sim 0.13 \mathrm{~cm}^{3} \cdot \mathrm{g}^{-1}$, see Table $\mathrm{S} 6$ and Figure $\mathrm{S} 12$, and from fluorescence data: $\mathrm{V} \sim 0.15 \mathrm{~cm}^{3} \cdot \mathrm{g}^{-1}$, see $\mathrm{SI}$ ) is within the 
range of its theoretical volume $\left(\mathrm{V} \sim 0.24 \mathrm{~cm}^{3} \cdot \mathrm{g}^{-1}\right.$, estimated by taking into account the volume of a $\mathrm{HOE}$ molecule under vacuum and its loading in the solid, see $\mathrm{SI}$ ). In fact, although the estimated HOE dimensions $(18.5 \times 4.1 \times 4.1 \AA)$ are slightly larger than the accessible windows of ZIF-8 $(\sim 3.6 \AA),{ }^{[20]}$ the potential HOE rearrangement and the flexibility of $\mathrm{ZIF}-8,{ }^{[21]}$ support the HOE location within the porosity, being consistent with SERS results.

Colloidal stability of the PMA- and non-PMA functionalized systems was evaluated in water and cell medium during one week by DLS (Figure 1G, Figure S13 and Table S7). The integrity of non-PMA samples was compromised in both media as reflected by the rapid decrease of $d_{h}(<5 h)$. Scanning electron microscopy (SEM) inspection of NC redispersed in water illustrates their "disintegration" (Figure S14). By simple visual inspection, one could also see how the characteristic turbidity of the NC gradually disappears within few hours (Figure S14). In contrast, $d_{h}$ of PMA samples remained stable, indicating that water or cell medium did not affect their integrity, which was also corroborated by SEM inspection of NC-PMA after dispersion for over one week in water (Figure S5).

To investigate the thermoplasmonic behaviour of the samples, non-PMA (in methanol, see Figure S15A) and the PMA (in water, see Figure 2A) systems, solutions at different concentration were irradiated during $1 \mathrm{~min}\left(7 \mathrm{~W} \cdot \mathrm{cm}^{-2}\right)$ with a NIR optical system (Figure S19). The bulk temperature of the solutions after NIR excitation was measured, showing the expected thermoplasmonic behaviour: the greater the concentration, the higher temperatures were achieved $\left(\sim 46^{\circ} \mathrm{C}-56^{\circ} \mathrm{C}\right)$. After irradiation, samples were precipitated and the $\mathrm{HOE}$ content (released) was quantified by fluorescence. In both non-PMA and PMA systems, the same trend was observed: irradiated samples showed a much higher HOE's release than the control ones, which were heated in a thermostatic batch $\left(1 \mathrm{~min}, 50^{\circ} \mathrm{C}\right)$, or nontreated samples (RT). To investigate the encapsulation efficiency of the NC-HOE-PMA system, HOE's releases were quantified in the irradiated $\left(1 \mathrm{~min}, 7 \mathrm{~W} \cdot \mathrm{cm}^{-2}\right)$ and non-irradiated (RT) control samples, at different time points: 0,8 and $24 \mathrm{~h}$ after irradiation. In the non-irradiated samples (RT), either in water (Figure 2B) or in phosphate buffered saline (PBS, Figure S16), HOE's releases were maintained negligible $(\leq 1 \%)$ over time, in contrast to the $\mathrm{NC}-\mathrm{HOE}$ system in methanol in which HOE's sustained release occurred, reaching 20\% HOE's release after 24 h (Figure S15). In the irradiated NC-HOE-PMA system in water or PBS, the initial HOE's release ( $t=0, \sim 30 \%$ of the maximum HOE loading) remained nearly constant over time. On the other hand, the NCHOE system in methanol showed a $10 \%$ HOE release after irradiation, which was increased due to the inherent sustained release of the non-PMA system in methanol (Figure S15).

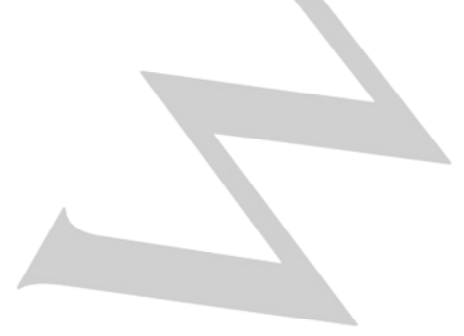

A

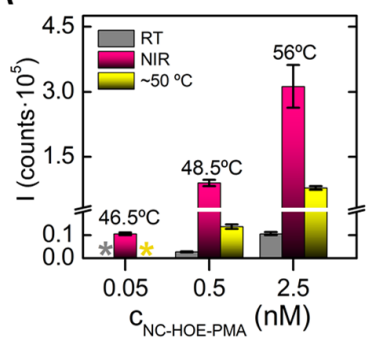

B

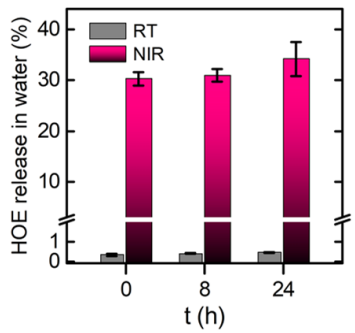

Figure 2. A) HOE's release in water by fluorescence (I) after NIR illumination ( $1 \mathrm{~min}, 7 \mathrm{~W} \cdot \mathrm{cm}^{-2}$ ) of the NC-HOE-PMA system (pink) at different concentrations (c); as controls, NC-HOE-PMA was heated in a thermostatic batch $\left(50{ }^{\circ} \mathrm{C}, 1 \mathrm{~min}\right.$, yellow) or non-heated (grey, room temperature RT); control samples at c $=0.05$ $\mathrm{nM}$ did not show any significant $\mathrm{HOE}$ 's release ( ${ }^{*}$ bellow the detection limit). B) HOE's release (\% of maximum HOE loading) in water for the NC-HOE-PMA system (pink, $\mathrm{c}=0.5 \mathrm{nM}$ ) immediately $(0 \mathrm{~h}), 8 \mathrm{~h}$ and $24 \mathrm{~h}$ after NIR illumination; as controls, non-treated equivalent samples (grey, RT) are shown (HOE's release $<0.5 \%$ )

HOE loaded, non-PMA and PMA NC systems were added to adherent cells (see SI). The viability of cells was similarly affected by both systems, showing the expected characteristic doseresponse behavior (2-300 nM NCs, Figure S13). The intracellular monitoring of cargo delivery upon illumination with a NIR light (785 $\mathrm{nm}, 3 \mathrm{~mW}$ ) was carried out by using SERS imaging with a confocal microscope. Figure 3A shows the SERS spectra and the $\mathrm{HOE}$ intensity (Figure $3 \mathrm{~B}$, ring breathing, $980 \mathrm{~cm}^{-1}$ ) registered in the mapping of cells (Figure $3 \mathrm{C}$ ) for both composites loaded with $\mathrm{HOE}$, with and without irradiation. No signal belonging to $\mathrm{HOE}$ was detected for $\mathrm{NC}-\mathrm{HOE}$. This is consistent with degradation of the ZIF-8 in water previously to the cell uptake. Conversely, in the case of NC-HOE-PMA, a clear HOE's signal was reordered in certain regions of the cell (likely in lysosomes) ${ }^{[22]}$ previous to the light illumination. In fact, after $8 \mathrm{~h}$ of irradiation, no HOE's signal could be discerned. However, the release kinetics clearly show that $\sim 75 \%$ of the $\mathrm{HOE}$ is released during the first $2 \mathrm{~h}$ of illumination. These results contrast with those obtained for the same material but without continuous illumination. At time 0 and after $8 \mathrm{~h}$ SERS spectra show clear HOE signals indicating that the integrity of the drug carrier was not compromised.

SERS data fully agree with equivalent studies by confocal microscopy (Figure 3D-F). NC-HOE-PMA showed accumulation (blue colored dots) in the vicinity of the nuclei, which did not present blue-staining due to the lack of HOE leaking (Figure 3E); in contrast, as expected from the stability studies and SERS imaging, the non-PMA system showed bright nucleus staining as consequence of the HOE leaking (that is, lack of integrity of the non-PMA system in cell medium, Figure 3D). Finally, NIR-induced HOE-release from the PMA system inside living cells was attempted. Cells containing NC-HOE-PMA were irradiated with the NIR illumination setup $\left(5 \mathrm{~min}, 7 \mathrm{~W} \cdot \mathrm{cm}^{-2}\right.$, beam diameter $\sim 1$ $\mathrm{cm}^{2}$ ) and samples were immediately inspected in the confocal microscope, which showed the HOE release, as can be concluded from the nuclei staining (Figure 3F).

In conclusion, our approach is an important step forward in the development of MOF-based intracellular vehicles for drug vectorization. We developed an aqueous stable (even when 
stored in cells) ZIF-8 based nanomaterial with combined thermoplasmonic and high drug-loading capabilities.
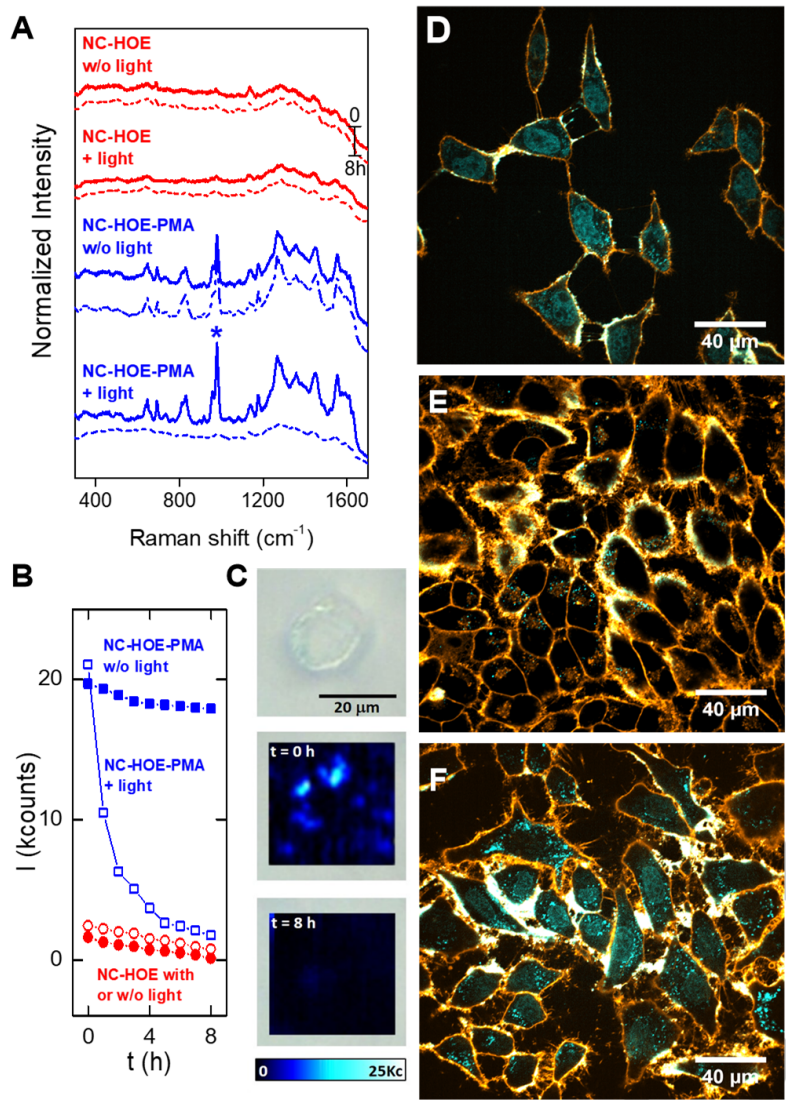

Figure 3. A) SERS spectra of the NCs after incubation with cells. Spectra kinetics were acquired with and without NIR illumination $(785 \mathrm{~nm})$; solid and dashed lines represent the time points $0 \mathrm{~h}$ and $8 \mathrm{~h}$, respectively. B) SERS intensity of the ring breathing of $\operatorname{HOE}\left(980 \mathrm{~cm}^{-1}\right)$ with time for both materials, with and without NIR light. C) Representative SERS imaging of a single cell incubated with NC-HOE-PMA. Optical image (top) and SERS images at time 0 (middle) and after $8 \mathrm{~h}$ of irradiation (down). D-F) Confocal microscopy image of cells incubated with NC-HOE (D), NC-HOE-PMA(E) and NC-HOE-PMA after NIR treatment $(F)$. In D-F, blue and orange color represent HOE and cell membrane staining (CellMask ${ }^{\mathrm{TM}}$ Deep Red), respectively; scale bar is $40 \mu \mathrm{m}$.

\section{Experimental Section}

Extended methods and characterization of the materials described in this communication (Figure S1-S23; Table S1-S7) can be found in the Supporting Information.

\section{Acknowledgements}

This work has received financial support from the MINECO-Spain (MAT2016-80266-R, MAT2015-74381-JIN, CTQ2017-88648R, ENE2016-79608-C2-1-R, CTQ2017-89588-R, RYC-2014-15039, RYC-2014-16962), the Xunta de Galicia (Centro singular de investigación de Galicia accreditation 2016-2019, ED431G/09,
Agrupación Estratégica de Materiales Action ED431E 2018/08), the Generalitat de Cataluña (2017SGR522, 2017SGR883, SLT002/16/00239), the URV (2017PFR-URV-B2-02), the German Research Society (DFG PA 794-21-1), and the European Union (European Regional Development Fund - ERDF, H2020MSCA-IF-2016 project 749667). MFNP acknowledges the CONACYT PhD fellowship program.

Keywords: MOF $\cdot$ ZIF-8 $\bullet$ thermoplasmonic $\cdot$ drug-release $\bullet$ nanocomposite

[1] G. Maurin, C. Serre, A. Cooper, G. Ferey, Chem. Soc. Rev. 2017, 46, 3104-3107.

[2] A. C. McKinlay, R. E. Morris, P. Horcajada, G. Férey, R. Gref, P. Couvreur, C. Serre, Angew. Chem. Int. Ed. 2010, 49, 6260-6266.

[3] K. S. Park, Z. Ni, A. P. Côté, J. Y. Choi, R. Huang, F. J. Uribe-Romo, H. K. Chae, M. O'Keeffe, O. M. Yaghi, Proc. Natl. Acad. Sci. U.S.A 2006, 103, 10186-10191.

[4] H. Nabipour, M. H. Sadr, G. R. Bardajee, New J. Chem. 2017, 41, 73647370.

[5] L. Yan, X. Chen, Z. Wang, X. Zhang, X. Zhu, M. Zhou, W. Chen, L. Huang V. A. L. Roy, P. K. N. Yu, G. Zhu, W. Zhang, ACS Appl. Mater. Interfaces 2017, 9, 32990-33000.

[6] X. Chen, R. Tong, Z. Shi, B. Yang, H. Liu, S. Ding, X. Wang, Q. Lei, J. Wu, W. Fang, ACS Appl. Mater. Interfaces 2018, 10, 2328-2337.

[7] H. Kaur, G. C. Mohanta, V. Gupta, D. Kukkar, S. Tyagi, J. Drug Deliv. Sci. Technol. 2017, 41, 106-112.

[8] X. Liu, Y. Li, Y. Ban, Y. Peng, H. Jin, H. Bux, L. Xu, J. Caro, W. Yang, Chem. Commun. 2013, 49, 9140-9142.

[9] H. Zhang, D. Liu, Y. Yao, B. Zhang, Y. S. Lin, J. Membr. Sci. 2015, 485, 103-111.

[10] M. Karimi, A. Ghasemi, P. Sahandi Zangabad, R. Rahighi, S. M. Moosavi Basri, H. Mirshekari, M. Amiri, Z. Shafaei Pishabad, A. Aslani, M. Bozorgomid, D. Ghosh, A. Beyzavi, A. Vaseghi, A. R. Aref, L. Haghani, S. Bahrami, M. R. Hamblin, Chem. Soc. Rev. 2016, 45, 1457-1501.

[11] G. Zheng, S. de Marchi, V. López-Puente, K. Sentosun, L. Polavarapu, I. Pérez-Juste, E. H. Hill, S. Bals, L. M. Liz-Marzán, I. Pastoriza-Santos, J. Pérez-Juste, Small 2016, 12, 3935-3943.

[12] Z. Wang, X. Tang, X. Wang, D. Yang, C. Yang, Y. Lou, J. Chen, N. He, Chem. Commun. 2016, 52, 12210-12213.

[13] L. He, M. Brasino, C. Mao, S. Cho, W. Park, A. P. Goodwin, J. N. Cha, Small 2017, 13, 1700504

[14] P. Hu, J. V. Morabito, C.-K. Tsung, ACS Catalysis 2014, 4, 4409-4419.

[15] J. Hühn, C. Carrillo-Carrion, M. G. Soliman, C. Pfeiffer, D. Valdeperez, A. Masood, I. Chakraborty, L. Zhu, M. Gallego, Z. Yue, M. Carril, N. Feliu, A. Escudero, A. M. Alkilany, B. Pelaz, P. del Pino, W. J. Parak, Chem. Mater. 2017, 29, 399-461.

[16] E. Polo, M. F. Navarro Poupard, L. Guerrini, P. Taboada, B. Pelaz, R. A. Alvarez-Puebla, P. del Pino, Nano Today 2018, 20, 58-73.

[17] C.-H. Kuo, Y. Tang, L.-Y. Chou, B. T. Sneed, C. N. Brodsky, Z. Zhao, C.K. Tsung, J. Am. Chem. Soc. 2012, 134, 14345-14348.

[18] J. Bucevičius, G. Lukinavičius, R. Gerasimaitè, Chemosensors 2018, 6 , 18.

[19] G. Kumari, K. Jayaramulu, T. K. Maji, C. Narayana, J. Phys. Chem. A 2013, 117, 11006-11012.

[20] A. Knebel, B. Geppert, K. Volgmann, D. I. Kolokolov, A. G. Stepanov, J. Twiefel, P. Heitjans, D. Volkmer, J. Caro, Science 2017, 358, 347-351.

[21] D. Fairen-Jimenez, S. A. Moggach, M. T. Wharmby, P. A. Wright, S. Parsons, T. Düren, J. Am. Chem. Soc. 2011, 133, 8900-8902.

[22] P. Rivera Gil, C. Vazquez-Vazquez, V. Giannini, M. P. Callao, W. J. Parak, M. A. Correa-Duarte, R. A. Alvarez-Puebla, Angew. Chem. Int. Ed. 2013, 52, 13694-13698. 
Entry for the Table of Contents (Please choose one layout)

Layout 1:

\section{COMMUNICATION}

A thermoresponsive carrier comprising gold nanostars coated with ZIF-8 stabilized with an amphiphilic polymer was developed for lighttriggered release of encapsulated cargo inside cells. The newly developed aqueous stable (even when stored in cells) nanocomposite combines thermoplasmonic and high drug-loading capabilities.

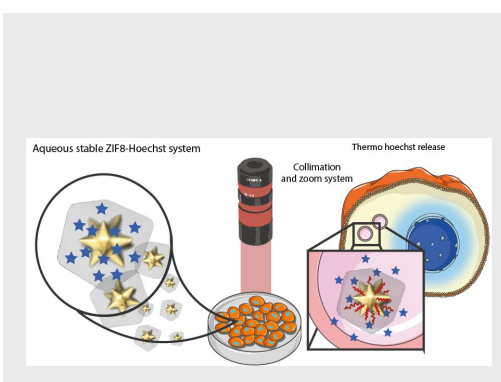

C. Carrillo-Carrión, R. Martínez, M. F. Navarro Poupard, B. Pelaz, E. Polo, A. Arenas-Vivo, A. Olgiati, P. Taboada, M. G. Soliman, Ú. Catalán, S. FernándezCastillejo, R. Solà, W. J. Parak, P. Horcajada, ${ }^{*}$ R. A. Alvarez-Puebla, ${ }^{*}$ and P. del Pino*

Page No. - Page No.

Aqueous Stable Gold Nanostar/ZIF-8 Nanocomposites for Light Triggered Release of Active Cargo Inside Living Cells 